\title{
Failure in evoking the trigeminal cardiac reflex by mandibular stretching in healthy volunteers
}

\author{
C. DE INNOCENTIII', C.G. CAPUTI', F. PINTO', S. QUINTILIANI', A. MECCARIELLO², \\ G. RENDA', M. DI NICOLA ${ }^{3}$, R. DE CATERINA', M. D'ATTILIO²
}

${ }^{7}$ Institute of Cardiology and Center of Excellence on Aging, Department of Neurosciences, Imaging and Clinical Sciences; ${ }^{2}$ Department of Medical, Oral and Biotechnological Sciences; ${ }^{3}$ Laboratory of Biostatistics, Department of Experimental and Clinical Sciences, G. d'Annunzio University, Chieti, Italy

\section{A B S T R A C T}

Stimulation of trigeminal sensory afferences has been reported to evoke hypotension and bradycardia, a phenomenon known as the trigeminal cardiac reflex. We attempted to evoke such a reflex through cycles of alternate mandibular stretching in healthy volunteers, as previously reported, for its possible therapeutic exploitation.

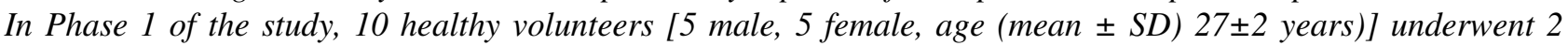
randomized sessions of automated monitoring, every 6 minutes, of systolic blood pressure (SBP), diastolic (D) BP, and heart rate (HR), with a one-week interval, either with mandibular stretching (12 minutes with a spring device fitted in the mouth), or nothing (control). Observation was prolonged for 180 minute after the end of the stretching. In Phase 2, 7 other volunteers (4 male and 3 female, age 24 1.3 years) repeated the protocol with a sampling interval of 2 minutes until the end of stretching.

Baseline levels of SBP, DBP and HR were similar in the test and control sessions. There was a progressive fall of $B P$ and $H R$ as a function of time during the test session. With stretching: SBP changed from 119.2 \pm 10.1 to $118.1 \pm 10.1$ to $115.8 \pm 10.5 \mathrm{mmHg}$, at baseline, end of stretching and 180 minutes after, respectively, $P<0.001$ at ANOVA for time effect). However, similar changes occurred in the control group: from $120.7 \pm 12.0$ to $120.8 \pm 12.0$ to $115.4 \pm 3.6 \mathrm{mmHg}$ at the same times, $P=0.822$ for group effect). In Phase 2, again we observed no significant changes for any of the parameters investigated as a function of treatment.

Despite attempts at maximum standardization of study condition and the use of operator-independent BP and HR measurements, we could not detect significant BP or HR effects of repeated mandibular stretching.

Key words

Trigeminal cardiac reflex $\bullet$ Gnawing $\bullet$ Mandibular stretching $\bullet$ Blood pressure $\bullet$ Heart rate

\section{Introduction}

The trigeminal cardiac reflex (TCR) is a brainstem reflex featuring the rapid occurrence of bradycardia, hypotension, apnea and gastric hypermotility during stimulation of trigeminal nerve branches. The reflex is a complex physiological response involving both the sympathetic and the parasympathetic nervous systems in response to various stimuli, such as craniofacial pain, the presence of fluids in the nasal cav- ity, and the mechanical stimulation of the ocular and periocular structures (Chowdhury et al., 2014;Gorini et al., 2009; Roberts et al., 1999). The reflex was first described by Krätschmer in 1870 in cats and rabbits (Blanc, 1991). In humans the TCR was first described by Schaller et al., highlighting that bradycardia and hypotension do not occur in patients treated with atropine, an M-acetylcholine receptor antagonist, suggesting the involvement of a vagal efferent pathway (Schaller B. et al., 1999). These authors described that 


\section{Phase 1}

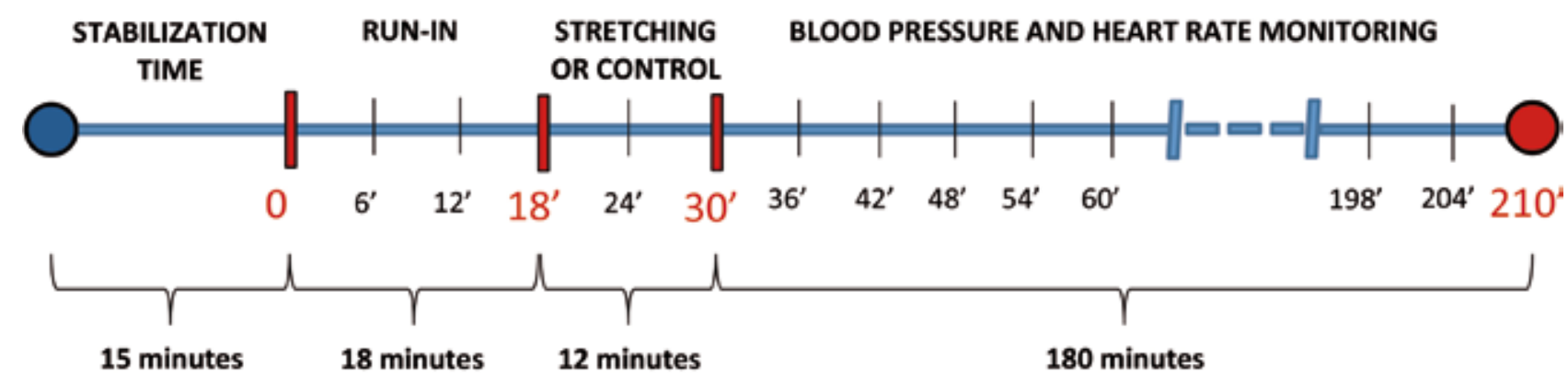

\section{Phase 2}

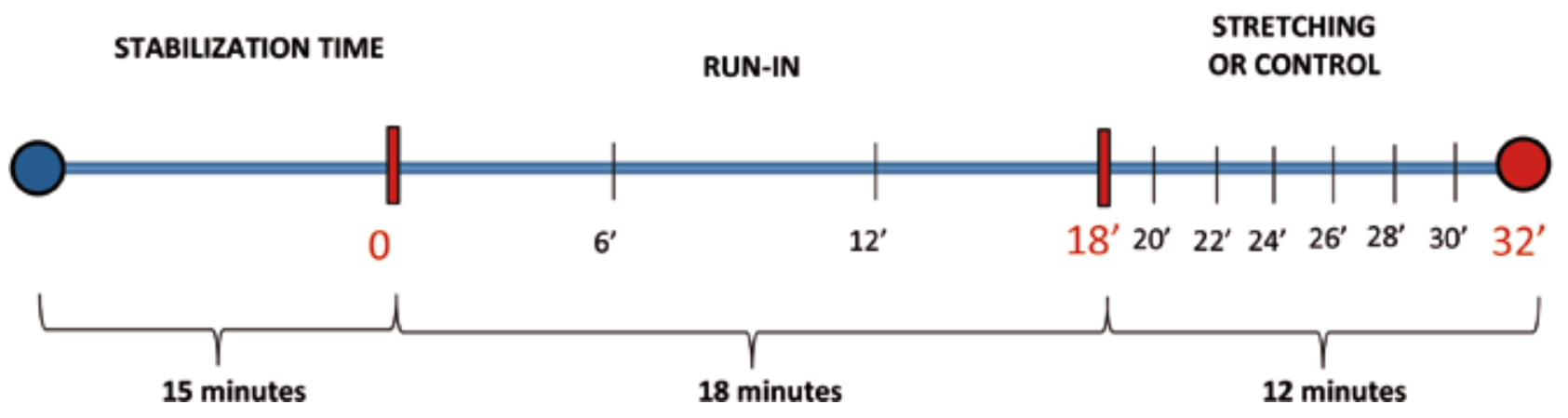

Fig. 1. - Timelines of Phase 1 (upper panel) and Phase 2 (lower panel) of the study.

sensory nerve fibers in the trigeminal nerve activate, through the Gasser ganglion, the sensitive nucleus of the trigeminal nerve, with a reflexion path through the short fibers of the reticular formation, reaching out the motor dorsal nucleus of the vagus. From here, cardio-inhibitory efferent fibers would reach the heart and the arterial/arteriolar vessels, causing bradycardia and hypotension (Chowdhury, Sandu, et al., 2014;Schaller B., Probst, et al., 1999).

Activation of the TCR occurs in disparate conditions including maxillary and dental manipulations, such as tooth extractions, maxillofacial surgery (Lubbers et al., 2010), and neurosurgical interventions (Schaller B., Probst, et al., 1999; Schaller B. J., 2007), as well as within the so called "diving reflex". This latter, elicited by cold water hitting the face of a scuba diver, and featuring bradycardia, peripheral vasoconstriction and the passage of plasma in the pulmonary alveoli, may have evolved to avoid alveolar collapse and counteract hypertension (McCulloch et al., 1999). The involvement of a vagal efferent pathway would be responsible for its fast occurrence and resolution, with a return to baseline values of heart rate soon at the end of stimulation. The rapid disappearance of the response is explained by the rapid breakdown of acetylcholine released during vagal stimulation (Blanc, 1991).

In 2012, Brunelli et al. have reported, contrary to the above-reported fast-waning responses, longlasting effects of mandibular stretching using a spring device placed between the upper and lower incisors in an experimental study in healthy volunteers (Brunelli et al., 2012), and this has been more recently confirmed in rats (Lapi et al., 2013).

The elicitation of a hypotensive response from mandibular stretching has potential therapeutic applications, especially if the hypotensive and heart rate-lowering response were to be protracted over time, as reported in the above-cited human study (Brunelli, Coppi, et al., 2012).

In order to further explore the therapeutic potential of the TCR, we attempted at reproducing such data and to standardize the stretching procedure. As a subordinate goal, we aimed at assessing the duration of the reflex responses over time after the end of mandibular stretching in healthy volunteers. This 
article details our failure to reproduce the reflex in a practically feasible fashion through mandibular stretching.

\section{Materials and methods}

\section{Aims and Subjects}

The primary objective of the study was to reproduce previous data in human volunteers (Brunelli, Coppi, et al., 2012) and to precisely assess the duration of the reflex responses after the end of mandibular stretching, for the possible practical exploitation of these findings. Because of the results accrued in this phase of the study, we undertook a second sub-study, to sample blood pressure and heart rate carefully at higher frequency during the stretching procedure.

In the first phase, 10 volunteers ( 5 male and 5 female) participated. In the second phase, we recruited other 7 subjects (4 male and 3 female).

We included young subjects (age range 20-30 years) of both sexes, in a good health status and not taking any medication. Intake of any psychoactive substance, including coffee, tea, and caffeine-containing drinks, tobacco or alcohol, was not permitted beyond $8 \mathrm{a}$ a.m. in the morning of the study. The study was conducted in the afternoon hours, between 3 p.m. and 7 p.m. between June 2013 and March 2014, in a dedicated room at the outpatient clinic of Chieti University Hospital. We obtained written informed consent from all participating subjects.

\section{Study design}

All subject underwent 2 study sessions, one with and one without a 12-minute mandibular stretching, in random order, at 7-day distance.

Mandibular stretching was obtained with a U-shaped folded steel spring device, modeled according to a previous report (Brunelli, Coppi, et al., 2012), and inserted between the upper and the lower medial incisor teeth. The degree of mouth opening, measured by the inter-incisival distance, was of $4 \mathrm{~cm}$, and in all subjects was less than their maximal active mouth opening. During the 12 minutes of mandibular stretching subject were asked to enact gnawing movements by compressing and relaxing the spring device, but avoiding muscle fatigue, as in a previous report (Brunelli, Coppi, et al., 2012). Two subjects underwent the test at the same time.

In Phase 1 (10 volunteers), BP and HR were recorded with an ambulatory blood pressure monitoring (ABPM) system (SpaceLabs model 90207, Issaquah, WA, USA). The rate of $\mathrm{BP}$ and HR sampling was standardized in order to have, after an initial 15-minute stabilization time, 4 automatic measurements taken at time $0,6,12$ and 18 minutes (Fig. 1A). Immediately after the baseline (time 0) sampling, one of the 2 daily volunteers simultaneously studied was submitted to the mandibular stretching for 12 minutes with the spring device. and then continued BP and HR monitoring every 6 minutes until 192 minutes. In parallel, the other volunteer was submitted to the HR and BP monitoring with the same sampling rate and for the same duration, but without mandibular stretching (Fig. 1A). During the test, subjects were allowed to comfortably sit in an armchair and allowed leisure reading. In the second study phase (7 volunteers), the interval between BP and HR measurements after an initial phase of stabilization was reduced to 2 minutes to better assess any short-term changes in these 2 parameters. For this phase we used an outpatient clinic automated blood pressure recorder (Laica Stabil-O-Graph, model MD6036, programmableat variance from the ABPM device-, for such frequent sampling (Fig. 1B)).

Each volunteer in both study phases was asked to undergo the alternative (control or stretching procedure) after exactly seven days.

\section{Statistical analysis}

Descriptive qualitative variables were summarized as frequencies and percentages, and quantitative variables as mean and standard deviation (SD). Variables were then plotted as mean and standard error of the mean (SEM). The occurrence of differences in $\mathrm{BP}$ and $\mathrm{HR}$ in each volunteer as a function of time (variable 1) and of the procedure administered (stretching or control, variable 2) was analyzed with a repeated-measure 2-factor analysis of variance (ANOVA). The Tukey post-hoc test was used to evaluate the time location of any statistically significant difference between the 2 time courses of $\mathrm{BP}$ and HR in each subject. A P value $<0.05$ was considered as statistically significant. 

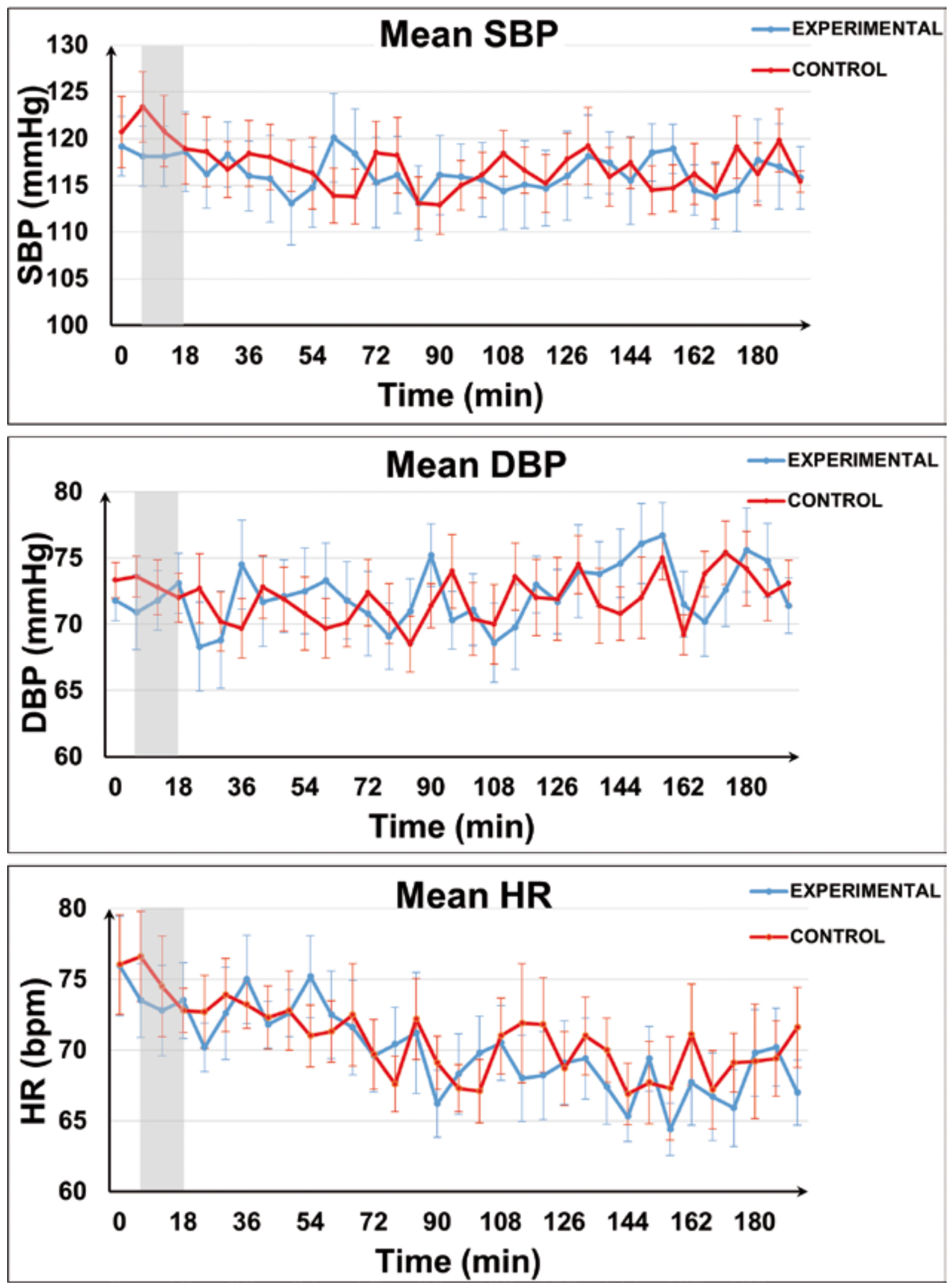

Fig. 2. - Time course of the systolic blood pressure (SBP), diastolic blood pressure (DBP) and heart rate (HR) in the experimental group (mandibular stretching) and the control group in Phase 1 of the study. Data at each time point are plotted as mean \pm SEM. For data analysis, see Table 2. 


\begin{tabular}{|l|c|}
\hline Table I. - Baseline characteristics of the population studied. & $27 \pm 2$ \\
\hline Phase 1 & $5 / 5$ \\
\hline Age (mean \pm SD) & $120.7 \pm 12.0$ \\
\hline Gender (males/females) & $73.3 \pm 4.2$ \\
\hline Baseline* SBP (mmHg, mean \pm SD) & $76.1 \pm 11.1$ \\
\hline Baseline* DBP (mmHg, mean \pm SD & \\
\hline Baseline* HR (beats/min, mean $\pm S D)$ & $24 \pm 1.3$ \\
\hline \multicolumn{2}{|c|}{4} \\
\hline Phase 2 & $4 / 3$ \\
\hline Age (mean \pm SD) & $124.8 \pm 12.5$ \\
\hline Gender (males/females) & $80.7 \pm 6.7$ \\
\hline Baseline* SBP (mmHg, mean \pm SD) & $74.5 \pm 13.2$ \\
\hline Baseline* DBP (mmHg, mean \pm SD) & \\
\hline Baseline* HR (beats/min, mean $\pm S D)$ & \\
\hline Abbreviations: SD = standard deviation; SBP = systolic blood pressure; DBP = diastolic blood pressure; HR = heart rate. \\
\hline *for each subject, the mean of 4 determinations was considered as the baseline value. \\
\hline
\end{tabular}

Statistical analyses were performed with the SPSS 11.0 software (SPSS Inc, Chicago, IL, USA) by an expert statistician (MDN), among the authors.

\section{Results}

Characteristics of the study subjects are detailed in Table I, and study results for systolic (S) BP, diastolic (D) BP and HR are reported in Table II.

Basal levels of SBP, DBP and HR were comparable between the 2 sets of measurements for the control and intervention sessions. For Phase 1, at baseline, SBP was $120.7 \pm 12.0 \mathrm{mmHg}$ and $119.2 \pm 10.1$ $\mathrm{mmHg}$; DBP was $73.3 \pm 4.2 \mathrm{mmHg}$ and $71.8 \pm 4.9$ $\mathrm{mmHg}$; HR was $76.0 \pm 4.8$ b.p.m. and $75.9 \pm 11.1$ b.p.m., for the control session and the mandibular stretching session, respectively (Table II).

A significant reduction in all 3 parameters as a function of time was observed in both the experimental and the control sessions. At ANOVA, for SBP: $\mathrm{P}$ $<0.001$; DBP: $\mathrm{P}<0.001$; HR: $\mathrm{P}=0.005$ (Table II and Figg. 2 and 3).

There was however no statistically significant difference in any of the 3 parameters between the 2 experimental sessions (Table II and Figg. 2 and 3). Interaction of time and treatment was not signifi- cant for any of the parameters analyzed. Analyzing only the 12 minutes of the actual intervention with the spring device $v s$ the control time, no differences in the time trends of SBP, DBP and HR were observed (Table II). Analyzing only the post-intervention time periods in both groups, no significant differences in any of the 3 parameters were found between the group having received the mandibular stretching $v s$ the control group (Table II). The time trends for all the 3 parameters investigated in the 2 experimental groups are represented in Fig. 2 as mean \pm SEM; and as individual values of the difference $(\Delta)$ between the control and the experimental group at each time point of sampling for each individual subject in Fig. 3.

In Phase 2 of the study, basal levels of SBP, DBP and HR were also comparable between the group assigned to the control and the group assigned to the intervention sessions. At baseline, SBP was $125.2 \pm 11.5 \mathrm{mmHg}$ and $124.8 \pm 12.5 \mathrm{mmHg}$; DBP was $80.7 \pm 6.2 \mathrm{mmHg}$ and $81.7 \pm 6.7 \mathrm{mmHg}$; HR was $74.7 \pm 13.1$ b.p.m. and $74.6 \pm 13.7$ b.p.m., for the control session and the mandibular stretching session, respectively.

There was this time no significant reduction in any of the 3 parameters as a function of time in both the experimental and the control sessions. At ANOVA for time effect: SBP: $\mathrm{P}=0.069$; DBP: $\mathrm{P}=0.889$; HR: $\mathrm{P}=0.053$. 

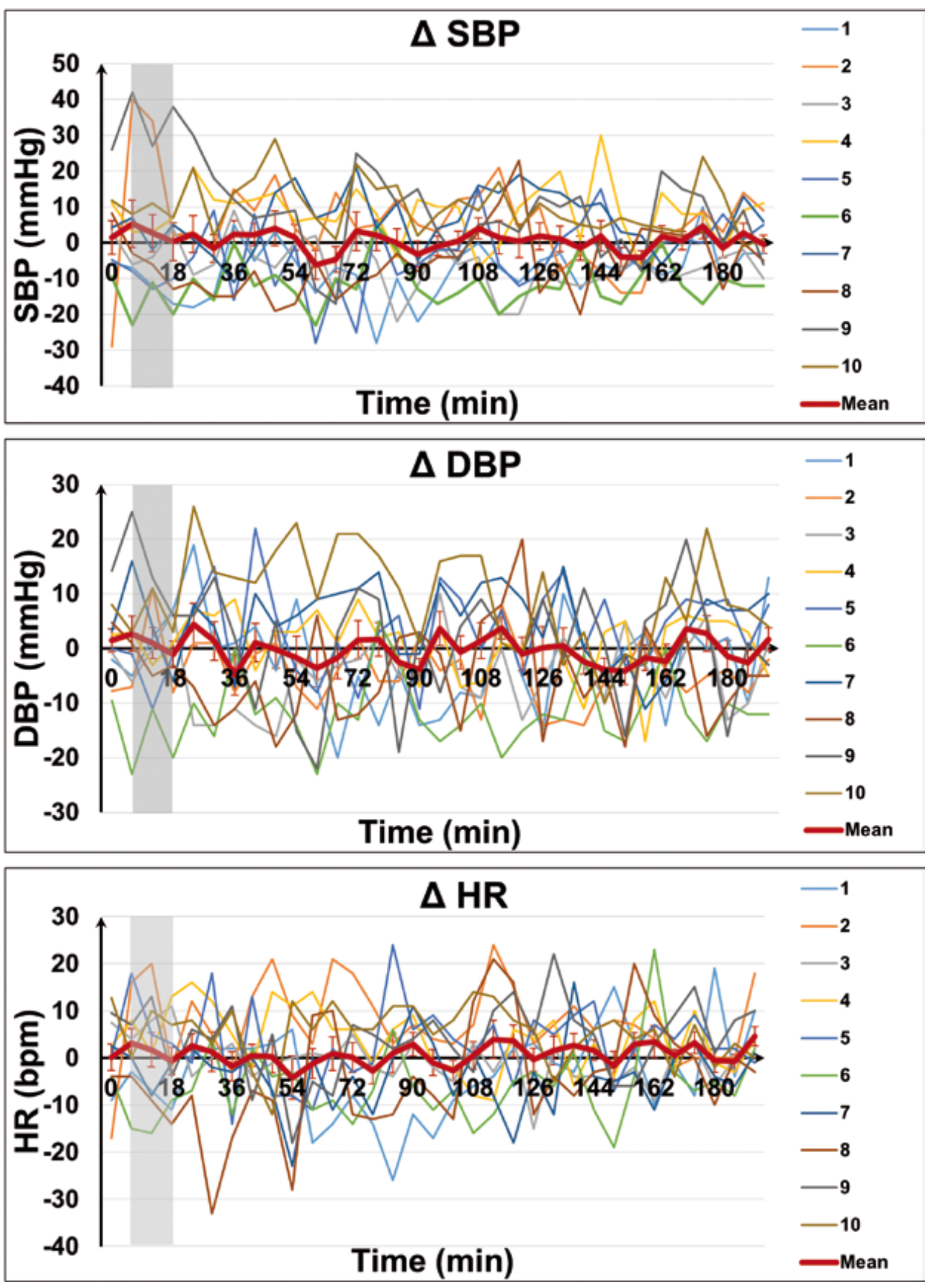

Fig. 3. - Time course of the absolute difference $(\Delta)$ in systolic blood pressure (SBP), diastolic blood pressure (DBP) and heart rate (HR) values in each study subject between the control and the experimental session in Phase 1 of the study. The average values of the differences $(\Delta)$ are also represented as a thick red line. For data analysis, see Table 2. 


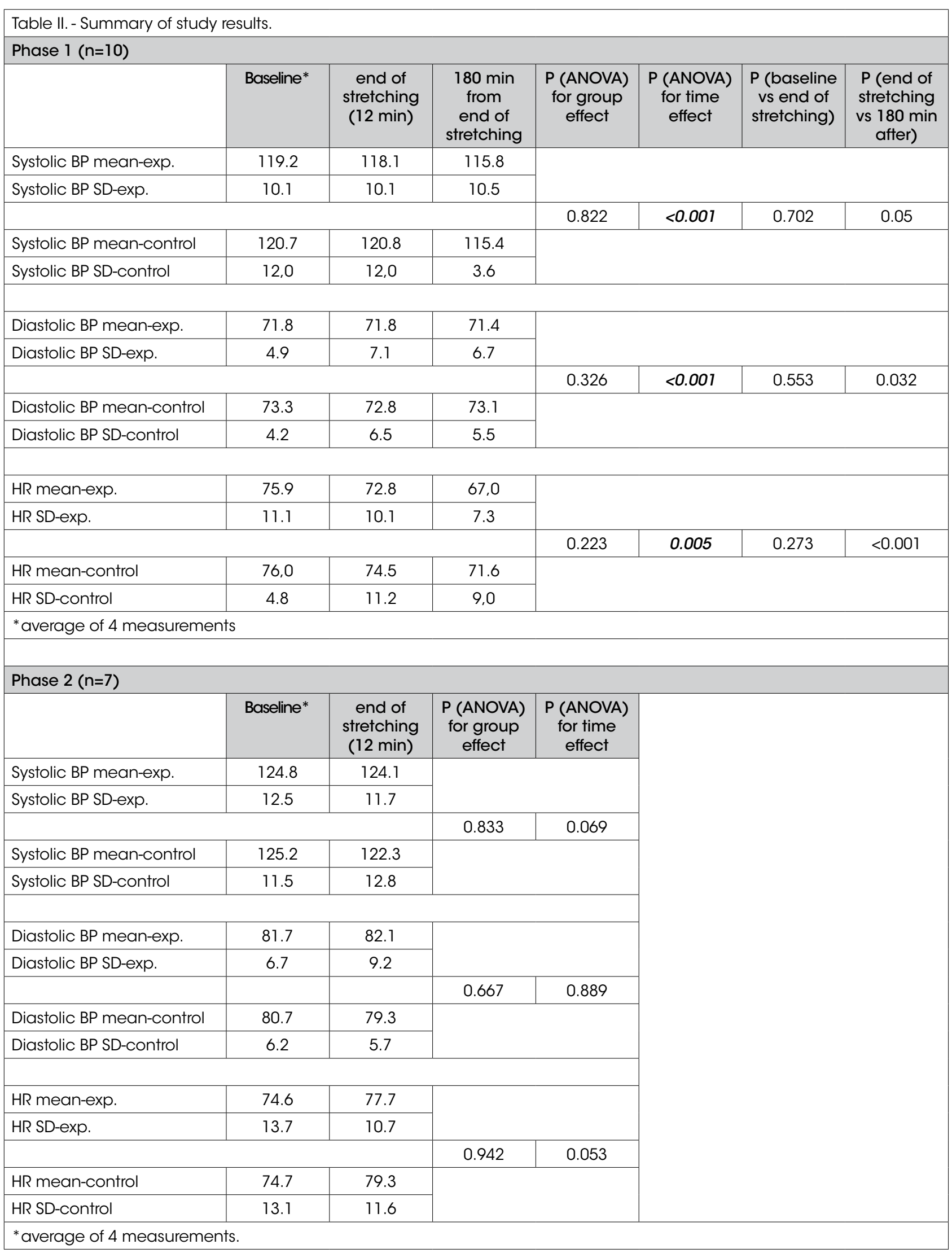



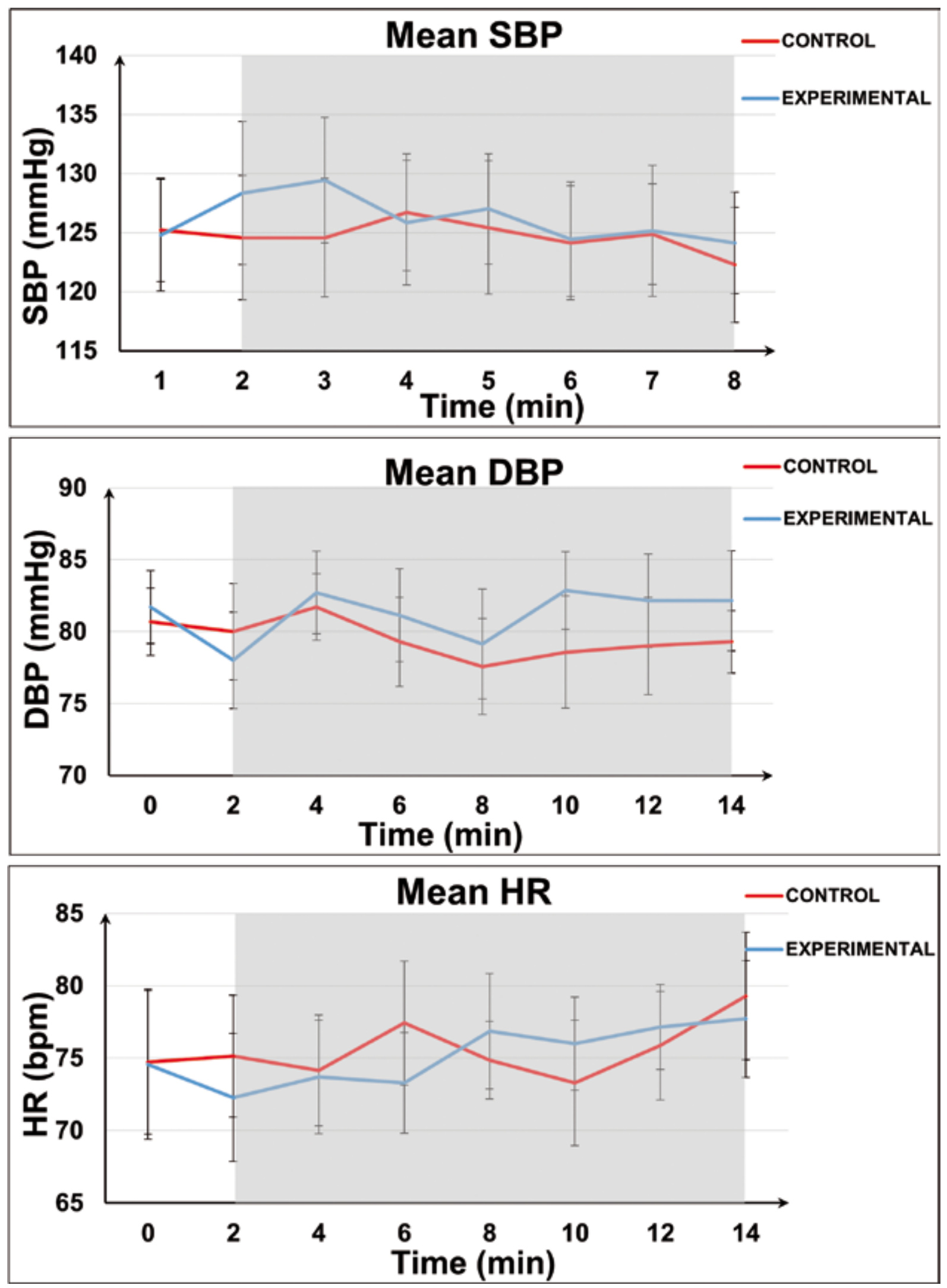

Fig. 4. - Time course of the systolic blood pressure (SBP), diastolic blood pressure (DBP) and heart rate (HR) in the experimental group (mandibular stretching) and the control group in Phase 2 of the study. Data at each time point are plotted as mean \pm SEM. For data analysis, see Table 2. 
Again, however, there was no statistically significant difference in any of the 3 parameters between the 2 experimental sessions. At ANOVA for group effect: SBP: $\mathrm{P}=0.833$; DBP: $\mathrm{P}=0.667$; HR: $\mathrm{P}=0.942$ (Table II). Interaction of time and treatment group was not significant for any of the parameters analyzed. The time trends for all the 3 parameters investigated in the 2 experimental groups are represented in Fig. 4 as mean \pm SEM; and as individual values of the $\Delta$ between the control and the experimental group at each time point of sampling for each individual subject in Fig. 5.

\section{Discussion}

The aim of the study was to demonstrate that mandibular stretching, obtained by means of a spring device applied for 12 minutes between the upper and lower incisors in association with active masticatory movements, induces a clinically significant and persistent reduction of $\mathrm{BP}$ and $\mathrm{HR}$, for a possible clinical exploitation of the underlying TCR. Indeed, if a reduction of BP could be demonstrated, it could lead to the use of such a non-pharmacological strategy. However data obtained clearly showed not even a trend to a differential outcome between the control and the experimental groups here studied. This is therefore a negative study, contradicting a previous report in human volunteers (Brunelli, Coppi, et al., 2012), despite attempting at reproducing similar experimental conditions.

Hypotension and bradycardia have been indeed observed (along with other symptoms, such as apnea and gastric hypermotility) after central or peripheral stimulation of any of the sensory branches of the trigeminal nerve. Clinically, these reflexes, collectively defined as TCR, have been reported to occur in disparate situations, including dental procedures - such as tooth extraction (Arakeri and Arali, 2010)_, craniofacial and neurosurgical procedures (Cha et al., 2002) — such as transphenoidal surgery for pituitary adenomas (Schaller B., 2005;Schaller B. J., 2007) and ponto-cerebellar tumor resections (Schaller B., Probst, et al., 1999).

Hypertension is the most common cardiovascular risk factor (Lewington et al., 2002), and there is ample documentation of substantial undertreatment in large segments of the hypertensive popula- tions (Mancia et al., 2013), justifying the need for alternative, non-pharmacological strategies. From a population health perspective, the mere demonstration of a reproducible technique that lowers blood pressure exploiting a physiological mechanism is of great medical importance, with relevant clinical implications, similar to attempts at evoking baroreceptor activation through carotid sinus pacing (Lohmeier and Iliescu, 2011), or renal denervation (Papademetriou et al., 2014). Independent reproduction of initial promising findings is therefore crucial, justifying the need of a report, such as ours, with essentially negative results.

Specifically, we first aimed at reproducing the findings of the study by Brunelli et al. (Brunelli, Coppi, et al., 2012), with a subordinate aim at characterizing the time duration of the response for a possible clinical exploitation. We tried to standardize BP and HR measurements with the use of an ABPM device, making the readings and data collection totally operator-independent. With this technique, we used a sampling interval of 6 minutes, the minimum available on our ABPM devices, to appreciate BP and HR changes with the highest reasonable accuracy. In addition, we prolonged the observation time to 180 minutes after the end of stretching, longer than the 80 minutes observation time in the previous study (Brunelli, Coppi, et al., 2012). We also elected to run a 15 minutes stabilization time preceding the application of the stimulus, to obtain 4 representative baseline measurements of BP and HR.

Against our original hypothesis, the ABPM results in the first 10 subjects did not reveal any substantial reduction of $\mathrm{BP}$ and $\mathrm{HR}$ after the application of the spring device compared with control conditions. While we observed a time-related trend to reduction in SBP, DBP and HR, probably as a function of the acquaintance of subjects to the study conditions, there was no difference in the experimental vs the control group over the 192 minutes of observations. Specifically, and contrary to our original hypothesis, no changes in BP were noticed either during or after the mandibular stretching. The study conducted by Brunelli et al. (Brunelli, Coppi, et al., 2012) is indeed the only one in the literature reporting a prolonged reduction in $\mathrm{BP}$ and $\mathrm{HR}$ rate following trigeminal stimulation in humans, thus allowing the hope of 

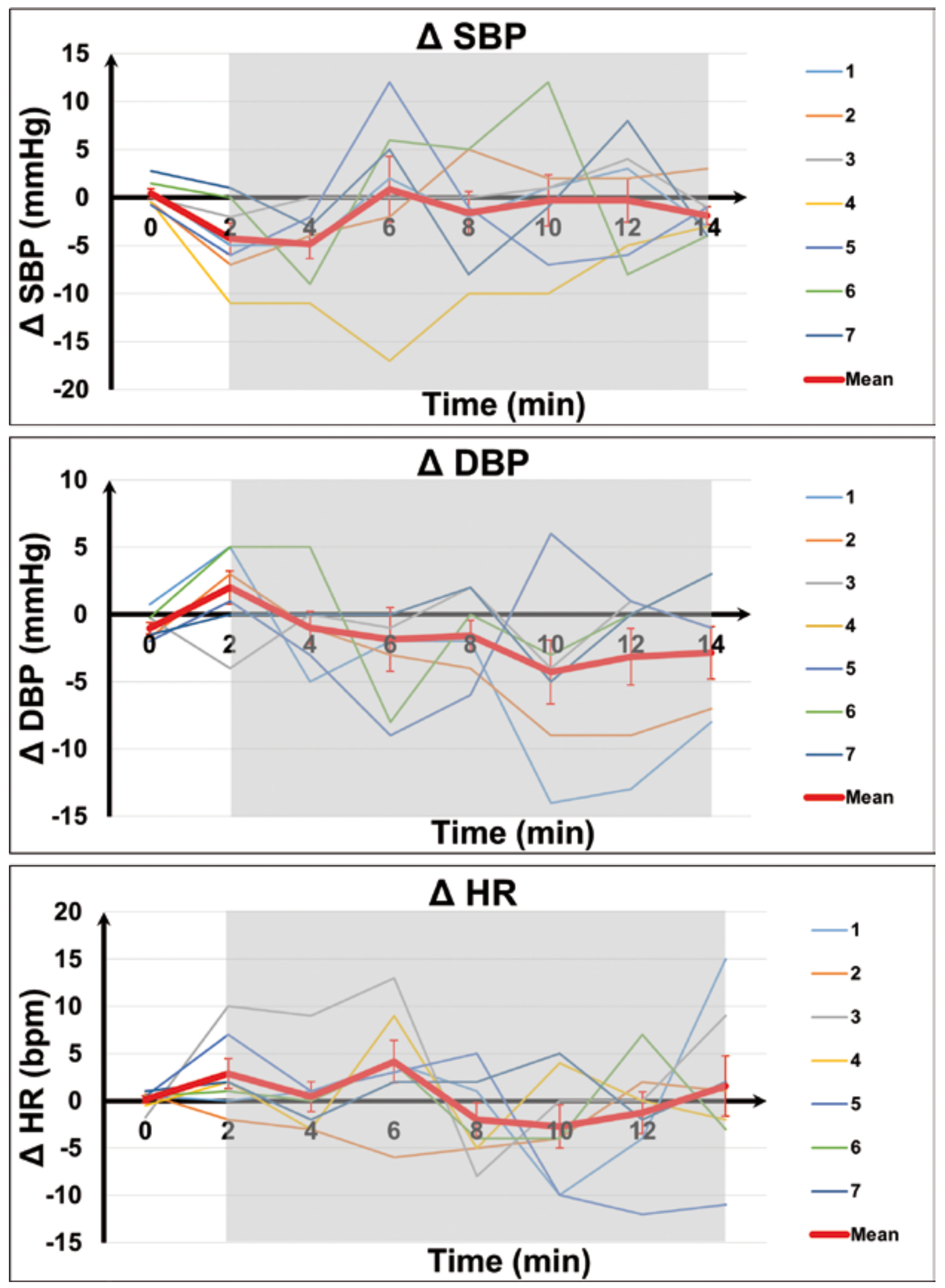

Fig. 5. - Time course of the absolute difference $(\Delta)$ in systolic blood pressure (SBP), diastolic blood pressure (DBP) and heart rate (HR) values in each study subject between the control and the experimental session in Phase 2 of the study. The average values of the differences $(\Delta)$ are also represented as a thick red line. For data analysis, see Table 2. 
translating this phenomenon into clinical practice. All other studies (Chowdhury, Sandu, et al., 2014), with the notable exception of another one in rats (Lapi, Colantuoni, et al., 2013), have shown that the TCR disappears with the termination of trigeminal stimulation, probably reflecting the rapid mechanism of hydrolysis of acetylcholine massively released by parasympathetic nerve endings after the stimulus.

In order to better investigate the possibility that there could be a reduction of $\mathrm{BP}$ and $\mathrm{HR}$ at least during the stretching phase, we extended the study to other 7 healthy volunteers, with characteristics similar to the first 10 subjects. In these, we collected the same parameters (BP and HR) during the control and intervention sessions, this time every 2 minutes. For this purpose, we used a different automated blood pressure monitor, allowing such a higher rate of sampling. Again, there were no significant differences in BP and HR immediately after application of the spring device compared with the control group, in all ways we analyzed the data.

The safest conclusion from this investigation is that mandibular stretching, in the way we applied it, does not consistently evoke trigeminal stimulation strong enough to elicit the cardio-inhibitory effect.

Probably the peripheral stimulation of the trigeminal nerve by the protocol we used is not sufficient to reproduce the TCR, at variance from that described after central stimulation of any of the sensory branches of the nerve (Gorini, Jameson, et al., 2009;Lubbers, Zweifel, et al., 2010;Roberts, Best, et al., 1999; Schaller B., Probst, et al., 1999;Schaller B., 2005). Such a conclusion applies to normotensive healthy volunteers, and it might well be that different effects can be found in hypertensive subjects. This would however need a separate investigation, not yet undertaken by us or others.

Reasons for the discrepancy of our findings with those of Brunelli et al. (Brunelli, Coppi, et al., 2012) are unclear. We tried to reproduce the same conditions as in that study, with a similar device. We allowed a reasonably long run-in phase in the study, during which volunteers could get acquainted with the protocol setting, and we totally relied upon automated BP monitoring to avoid any operator-related bias. In the Phase 1 of the study we prolonged the observation time after the stretching much beyond the 80 minutes of the previously referred study (Brunelli, Coppi, et al.,
2012), to our 180 minutes. In Phase 2, to further focus on the induction phase of the reflex, we increased the rate of BP and HR sampling to 2 per minute. None of such approaches yielded the expected results. A possibility remains that the extent of mandibular stretching in our study was different from that of the previously referred study (Brunelli, Coppi, et al., 2012), despite the use of a similar spring device. A different (greater) extent of mandibular stretching by Brunelli et al. might have induced stimulation of different type of fibers, as it is known that the sensory trigeminal nerve fibers are composed predominantly of unmyelinated $\mathrm{C}$ or myelinated small A- $\delta$ fibers (Schaller B., 2004). This raises the possibility that in the setting by Brunelli et al. nociceptors, other than mechanoceptors, were activated during submaximal mouth opening. Such considerations remain speculative at the moment.

\section{Conclusions}

The reproducibility of the TCR in humans by mandibular stretching through mandibular extension and gnawing movements in humans is questionable. Despite the standardization of the stimulus and of data collection, we failed to demonstrate any significant effects on BP and HR. Clarification of better physiological ways to elicit the TCR in humans awaits further studies.

\section{References}

Arakeri G., Arali V. A new hypothesis of cause of syncope: trigeminocardiac reflex during extraction of teeth. Med Hypotheses, 74: 248-251, 2010.

Blanc V.F. Trigeminocardiac reflexes. Can J Anaesth, 38: 696-699, 1991.

Brunelli M., Coppi E., Tonlorenzi D., Del Seppia C., Lapi D., Colantuoni A., Scuri R., Ghione S. Prolonged hypotensive and bradycardic effects of passive mandibular extension: evidence in normal volunteers. Arch Ital Biol, 150: 231-237, 2012.

Cha S.T., Eby J.B., Katzen J.T., Shahinian H.K. Trigeminocardiac reflex: a unique case of recurrent asystole during bilateral trigeminal sensory root rhizotomy. Journal of cranio-maxillo-facial surgery: official publication of the European Association for Cranio-Maxillo-Facial Surgery, 30: 108-111, 2002. 
Chowdhury T., Sandu N., Sadr-Eshkevari P., Meuwly C., Schaller B. Trigeminocardiac reflex: current trends. Expert Rev Cardiovasc Ther, 12: 9-11, 2014.

Gorini C., Jameson H.S., Mendelowitz D. Serotonergic modulation of the trigeminocardiac reflex neurotransmission to cardiac vagal neurons in the nucleus ambiguus. J Neurophysiol, 102: 1443-1450, 2009.

Lapi D., Colantuoni A., Del Seppia C., Ghione S., Tonlorenzi D., Brunelli M., Scuri R. Persistent effects after trigeminal nerve proprioceptive stimulation by mandibular extension on rat blood pressure, heart rate and pial microcirculation. Arch Ital Biol, 151: 11-23, 2013.

Lewington S., Clarke R., Qizilbash N., Peto R., Collins R. Age-specific relevance of usual blood pressure to vascular mortality: a meta-analysis of individual data for one million adults in 61 prospective studies. Lancet, 360: 1903-1913, 2002.

Lohmeier T.E., Iliescu R. Chronic lowering of blood pressure by carotid baroreflex activation: mechanisms and potential for hypertension therapy. Hypertension, 57: 880-886, 2011.

Lubbers H.T., Zweifel D., Gratz K.W., Kruse A. Classification of potential risk factors for trigeminocardiac reflex in craniomaxillofacial surgery. $J$ Oral Maxillofac Surg, 68: 1317-1321, 2010.

Mancia G., Fagard R., Narkiewicz K., Redon J., Zanchetti A., Bohm M., Christiaens T., Cifkova R., De Backer G., Dominiczak A., Galderisi M., Grobbee D.E., Jaarsma T., Kirchhof P., Kjeldsen S.E., Laurent S., Manolis A.J., Nilsson P.M., Ruilope L.M., Schmieder R.E., Sirnes P.A., Sleight P., Viigimaa M., Waeber B., Zannad F., Burnier M., Ambrosioni E., Caufield M., Coca A., Olsen M.H., Tsioufis C., van de Borne P., Zamorano J.L., Achenbach S., Baumgartner H., Bax J.J., Bueno H., Dean V., Deaton C., Erol C., Ferrari R., Hasdai D., Hoes A.W., Knuuti J., Kolh P., Lancellotti P., Linhart A., Nihoyannopoulos
P., Piepoli M.F., Ponikowski P., Tamargo J.L., Tendera M., Torbicki A., Wijns W., Windecker S., Clement D.L., Gillebert T.C., Rosei E.A., Anker S.D., Bauersachs J., Hitij J.B., Caulfield M., De Buyzere M., De Geest S., Derumeaux G.A., Erdine S., Farsang C., Funck-Brentano C., Gerc V., Germano G., Gielen S., Haller H., Jordan J., Kahan T., Komajda M., Lovic D., Mahrholdt H., Ostergren J., Parati G., Perk J., Polonia J., Popescu B.A., Reiner Z., Ryden L., Sirenko Y., Stanton A., Struijker-Boudier H., Vlachopoulos C., Volpe M., Wood D.A. 2013 ESH/ESC guidelines for the management of arterial hypertension: the Task Force for the Management of Arterial Hypertension of the European Society of Hypertension (ESH) and of the European Society of Cardiology (ESC). Eur Heart J, 34: 2159-2219, 2013.

McCulloch P.F., Faber K.M., Panneton W.M. Electrical stimulation of the anterior ethmoidal nerve produces the diving response. Brain Res, 830: 24-31, 1999.

Papademetriou V., Rashidi A.A., Tsioufis C., Doumas M. Renal nerve ablation for resistant hypertension: how did we get here, present status, and future directions. Circulation, 129: 1440-1451, 2014.

Roberts R.S., BestJ.A., Shapiro R.D. Trigeminocardiac reflex during temporomandibular joint arthroscopy: report of a case. J Oral Maxillofac Surg, 57: 854-856, 1999.

Schaller B., Probst R., Strebel S., Gratzl O. Trigeminocardiac reflex during surgery in the cerebellopontine angle. J Neurosurg, 90: 215-220, 1999.

Schaller B. Trigeminocardiac reflex. A clinical phenomenon or a new physiological entity? J Neurol, 251: 658-665, 2004.

Schaller B. Trigemino-cardiac reflex during transsphenoidal surgery for pituitary adenomas. Clin Neurol Neurosurg, 107: 468-474, 2005.

Schaller B.J. Trigeminocardiac reflex. J Neurosurg, 107: 243; author reply 243-244, 2007. 